\title{
Becke-Johnson-type exchange potential for two-dimensional systems
}

\author{
S. Pittalis, ${ }^{1,2,3, \text {, }}$ E. Räsänen, ${ }^{4, \text { 田 and C. R. Proetto }}{ }^{1,2, \text { 团 }}$ \\ ${ }^{1}$ Institut für Theoretische Physik, Freie Universität Berlin, Arnimallee 14, D-14195 Berlin, Germany \\ ${ }^{2}$ European Theoretical Spectroscopy Facility (ETSF) \\ ${ }^{3}$ Department of Physics and Astronomy, University of Missouri, Columbia, Missouri 65211, USA \\ ${ }^{4}$ Nanoscience Center, Department of Physics, University of Jyväskylä, FI-40014 Jyväskylä, Finland
}

(Dated: November 4, 2018)

\begin{abstract}
We extend the Becke-Johnson approximation [J. Chem. Phys. 124, 221101 (2006)] of the exchange potential to two dimensions. We prove and demonstrate that a direct extension of the underlying formalism may lead to divergent behavior of the potential. We derive a cure to the approach by enforcing the gauge invariance and correct asymptotic behavior of the exchange potential. The procedure leads to an approximation which is shown, in various quasi-two-dimensional test systems, to be very accurate in comparison with the exact exchange potential, and thus a considerable improvement over the commonly applied local-density approximation.

PACS numbers: 31.15.E-; 71.15.Mb; 73.21.La
\end{abstract}

\section{INTRODUCTION}

The advent of density-functional theory 1.2 (DFT) was followed by the development of approximate functionals for the exchange and correlation energy of many-electron systems. Significant advances were achieved beyond the local-density approximation (LDA) by generalizedgradient approximations, orbital functionals, and hybrid functionals $\stackrel{\underline{3}}{\underline{\underline{n}}}$ Those efforts focused almost solely on three-dimensional systems. However, already since the 1970s systems of reduced dimensionality became of great relevance in solid state physics. The present nanodevices consist of a large variety of low-dimensional systems, where the many-body effects of interacting electrons need to be addressed. Of particular interest are two-dimensional (2D) structures including, e.g., semiconductor layers and surfaces, quantum Hall systems, and various types of quantum dots $\underline{\underline{4}}$

Previous studies have shown that density functionals developed particularly for 3D fail when applied to quasi$2 \mathrm{D}$ systems $\stackrel{5,6,7}{ }$ By "quasi-2D" we mean physical systems whose main building block is the quasi-2D electron gas. This class comprises most systems listed in the previous paragraph and they can be reliably treated by a "pure" 2D approach, i.e., on a 2D grid. The physical reason for this is that the confinement potential along the growth direction (say $z$ ), is typically much stronger than in the two other spatial dimensions. This results in a quantum mechanical supression of the degrees of freedom along the $z$ direction, and the system becomes effectively $2 \mathrm{D}$. Then, the influence of the surrounding host material is dealt within the effective-mass approximation manifesting itself as an effective mass and a dielectric constant in the 2D Hamiltonian.

Within the DFT approach, 2D many-electron problems are most commonly treated using the 2D-LDA exchange functional $\mathrm{l}^{8}$ combined with the $2 \mathrm{D}-\mathrm{LDA}$ correlation parametrized first by Tanatar and Ceperley ${ }^{\underline{9}}$ and later, for the complete range of collinear spin polarization, by Attaccalite and co-workers $\stackrel{10}{2}$ Despite the rela- tively good performance of LDA with respect to, e.g., quantum Monte Carlo calculations $\frac{11}{1}$ in semiconductor quantum dots, there is a clear lack of accurate $2 \mathrm{D}$ density functionals to deal with diverse situations, especially in the strongly correlated regime. Only recently, the transition from $3 \mathrm{D}$ to $2 \mathrm{D}$ has been analyzed, $\stackrel{12}{\frac{1}{2}}$ and exchange-correlation energy functionals specially tailored for the 2D world have been proposed. $13,14,15,16,17,18,19,20$ Also very recently, a DFT framework specially tailored for strongly correlated electrons has been developed and successfully tested for few-electron quantum dots. $\underline{\underline{21}}$

In the present work, we aim at moving another step along the direction from 3D to 2D. We focus on approximations for the exchange potential, where our aim is to achieve an accuracy comparable to the exact result - available from the optimized-effective-potential (OEP) $\operatorname{method}^{22,23}$ or its approximations such as the KriegerLie-Iafrate (KLI) potential24 - yet simplifying the computational effort. We are able to find such an approximation by (i) extending the framework of the well-known Becke-Johnson potential ${ }^{25}$ to $2 \mathrm{D}$, and (ii) requiring the potential to satisfy the gauge invariance and correct asymptotic behavior. Our spin- and current-dependent exchange potential is indeed well comparable to the KLI and superior to the LDA in 2D quantum dots with varying sizes and varying external magnetic fields.

\section{FORMALISM}

\section{A. Spin-density functional theory}

Considering open-shell systems, (collinear) spin-DFT (SDFT) formalism ${ }^{26}$ is one of the most useful. In this case, the total energy $E$ of a $2 \mathrm{D}$ system of interacting electrons is a functional of the two spin densities, $\rho_{\sigma}(\mathbf{r})$ 
with $\sigma=\uparrow, \downarrow$ :

$$
\begin{aligned}
& E\left[\rho_{\uparrow}, \rho_{\downarrow}\right]=T_{s}\left[\rho_{\uparrow}, \rho_{\downarrow}\right] \\
& \quad+\int d^{2} r v_{0 \sigma}(\mathbf{r}) \rho(\mathbf{r})+U[\rho]+E_{x c}\left[\rho_{\uparrow}, \rho_{\downarrow}\right],
\end{aligned}
$$

where

$$
T_{s}\left[\rho_{\uparrow}, \rho_{\downarrow}\right]=\sum_{\sigma=\uparrow, \downarrow} \sum_{j}^{N_{\sigma}} \int d^{2} r \varphi_{j \sigma}^{*}(\mathbf{r})\left(-\frac{\nabla^{2}}{2}\right) \varphi_{j \sigma}(\mathbf{r}),
$$

is the non-interacting kinetic energy, the sum runs over occupied states, and $N_{\sigma}$ is the number of electrons with spin $\sigma$. Hartree atomic units (a.u.) are used, unless stated otherwise. Above, $v_{0 \sigma}(\mathbf{r})$ is the spin-dependent external potential. The classical electrostatic (Hartree) interaction energy is given by

$$
U[\rho]=\frac{1}{2} \int d^{2} r \int d^{2} r^{\prime} \frac{\rho(\mathbf{r}) \rho\left(\mathbf{r}^{\prime}\right)}{\left|\mathbf{r}-\mathbf{r}^{\prime}\right|}
$$

where

$$
\rho(\mathbf{r})=\rho_{\uparrow}(\mathbf{r})+\rho_{\downarrow}(\mathbf{r})
$$

is the total electronic density, which is the sum of the spin densities

$$
\rho_{\sigma}(\mathbf{r})=\sum_{j=1}^{N_{\sigma}}\left|\varphi_{j \sigma}(\mathbf{r})\right|^{2}
$$

with the sum running over occupied orbitals. Besides, $E_{x c}\left[\rho_{\uparrow}, \rho_{\downarrow}\right]$ is the exchange-correlation energy functional, which in practice needs to be approximated. The singleparticle orbitals $\varphi_{j \sigma}(\mathbf{r})$ in Eq. (2) are solutions of the Kohn-Sham (KS) equation ${ }^{27}$

$$
\left(-\frac{\nabla^{2}}{2}+v_{s \sigma}(\mathbf{r})\right) \varphi_{j \sigma}(\mathbf{r})=\varepsilon_{j \sigma} \varphi_{j \sigma}(\mathbf{r})
$$

where $j$ is a collective index for the one-electron quantum numbers, except for the spin. The effective single particle potential for spin $\sigma$ is given by

$$
v_{s \sigma}(\mathbf{r})=v_{0 \sigma}(\mathbf{r})+v_{\mathrm{H}}(\mathbf{r})+v_{x c \sigma}(\mathbf{r})
$$

with the Hartree potential

$$
v_{\mathrm{H}}(\mathbf{r})=\int d^{2} r^{\prime} \frac{\rho\left(\mathbf{r}^{\prime}\right)}{\left|\mathbf{r}-\mathbf{r}^{\prime}\right|}
$$

and the exchange-correlation potential

$$
v_{x c \sigma}(\mathbf{r})=\frac{\delta E_{x c}\left[\rho_{\uparrow}, \rho_{\downarrow}\right]}{\delta \rho_{\sigma}(\mathbf{r})}
$$

\section{B. Optimized-effective-potential method}

In the following, we restrict ourselves to orbital functionals. A standard example of this type of functionals is the exchange energy functional of the form

$$
E_{x}\left[\rho_{\uparrow}, \rho \downarrow\right]=-\frac{1}{2} \sum_{\sigma=\uparrow, \downarrow} \sum_{j, k=1}^{N_{\sigma}} \int d^{2} r \int d^{2} r^{\prime} \frac{\varphi_{j \sigma}^{*}(\mathbf{r}) \varphi_{k \sigma}^{*}\left(\mathbf{r}^{\prime}\right) \varphi_{j \sigma}\left(\mathbf{r}^{\prime}\right) \varphi_{k \sigma}(\mathbf{r})}{\left|\mathbf{r}-\mathbf{r}^{\prime}\right|},
$$

which resembles the Fock term of Hartree-Fock theory, here evaluated with KS orbitals. This is the so-called exact-exchange (EXX) energy functional. It should be noted that $E_{x}\left[\rho_{\uparrow}, \rho_{\downarrow}\right]$ is an implicit functional of the spin densities $\rho_{\sigma}(\mathbf{r})$. Of course, correlation can be considered as well in a similar framework. In this kind of functionals the calculation of the exchange-correlation potential requires the OEP method ${ }^{22,23}$ For a review of the method the reader is referred to Refs. 28, 29,30. Restricting ourselves to the EXX case, the OEP method leads to an integral equation for the exchange potential, which can be written in compact form as

$$
\sum_{j=1}^{N_{\sigma}}\left(\psi_{j \sigma}^{*}(\mathbf{r}) \varphi_{j \sigma}(\mathbf{r})+\text { c.c. }\right)=0,
$$

where the so-called orbital shifts are defined as

$$
\psi_{j \sigma}^{*}(\mathbf{r})=\int d^{2} r^{\prime} \varphi_{j \sigma}^{*}\left(\mathbf{r}^{\prime}\right)\left[v_{x \sigma}\left(\mathbf{r}^{\prime}\right)-u_{x j \sigma}\left(\mathbf{r}^{\prime}\right)\right] G_{j \sigma}^{S}\left(\mathbf{r}^{\prime}, \mathbf{r}\right) .
$$

Here $G_{j \sigma}^{S}$ is the Green function of the KS system,

$$
G_{j \sigma}^{S}\left(\mathbf{r}^{\prime}, \mathbf{r}\right)=\sum_{\substack{k=1 \\ \varepsilon_{k \sigma} \neq \varepsilon_{j \sigma}}}^{\infty} \frac{\varphi_{k \sigma}^{*}\left(\mathbf{r}^{\prime}\right) \varphi_{k \sigma}(\mathbf{r})}{\varepsilon_{j \sigma}-\varepsilon_{k \sigma}}
$$

and

$$
u_{x j \sigma}(\mathbf{r})=\frac{1}{\varphi_{j \sigma}^{*}(\mathbf{r})} \frac{\delta E_{x}}{\delta \varphi_{j \sigma}(\mathbf{r})}
$$

In a series of steps, $, 30,31$ the OEP equation as given in Eq. (11) can be transformed to 


$$
v_{x \sigma}(\mathbf{r})=\frac{1}{2 \rho_{\sigma}(\mathbf{r})} \sum_{j=1}^{N_{\sigma}}\left[\left|\varphi_{j \sigma}(\mathbf{r})\right|^{2}\left(u_{x j \sigma}(\mathbf{r})+\left(\bar{v}_{x j \sigma}-\bar{u}_{x j \sigma}\right)\right)-\nabla \cdot\left(\psi_{j \sigma}^{*}(\mathbf{r}) \nabla \varphi_{j \sigma}(\mathbf{r})\right)\right]+\text { c.c. }
$$

where

$$
\bar{v}_{x j \sigma}=\int d^{2} r \varphi_{j \sigma}^{*}(\mathbf{r}) v_{x \sigma}(\mathbf{r}) \varphi_{j \sigma}(\mathbf{r})
$$

and

$$
\bar{u}_{x j \sigma}=\int d^{2} r \varphi_{j \sigma}^{*}(\mathbf{r}) u_{x j \sigma}(\mathbf{r}) \varphi_{j \sigma}(\mathbf{r}) .
$$

The OEP equations can be solved iteratively, simultaneously with the corresponding KS equations, in a selfconsistent fashion.

Due to the presence of the unoccupied KS orbitals in the definition of the orbital shifts [see Eqs. (12) and (13)], the full numerical solution of the OEP integral equation is nontrivial. Of course, one may take advantage by specifying it for a particular kind of systems. In the original paper, $\stackrel{23}{r}$ solutions were presented for atomic systems with spherical symmetry. Much later, the OEP equation has also been solved for systems with lower symmetry such as molecules,,$\frac{32,33}{,}$ solids,,$\frac{34}{r}$ metallic surfaces,$\stackrel{35}{=}$ and quasi two-dimensional electron gases at the interface of two different semiconductors $\stackrel{20}{\underline{2}}$ In addition, an iterative algorithm for the solution of the OEP equation based on the orbital shifts has been implemented ${ }^{36.37}$ Recently, progress has been made in studying physically relevant examples of non-collinear magnetism, $, 38,39$ and open-shell systems in the relativistic limit $\underline{40,41}$ On the other hand, one may approximate the full OEP in order to save computational effort. Along this line, the KLI approach ${ }^{24}$ has turned out to be rather accurate in many situations. In the KLI approximation, the terms containing the orbital shifts on the r.h.s. of Eq. (15) are neglected completely $\underline{30}$

For the analysis which follows in the next sections, we may rewrite Eq. (15) as

$$
\begin{aligned}
v_{x \sigma}(\mathbf{r}) & =v_{x \sigma}^{\mathrm{SL}}(\mathbf{r})+\Delta v_{x \sigma}^{\mathrm{KLI}}(\mathbf{r})+\Delta v_{x \sigma}^{\mathrm{OS}}(\mathbf{r}) \\
& =v_{x \sigma}^{\mathrm{SL}}(\mathbf{r})+\Delta v_{x \sigma}^{\mathrm{OEP}}(\mathbf{r})
\end{aligned}
$$

where

$$
v_{x \sigma}^{\mathrm{SL}}(\mathbf{r})=\frac{1}{2 \rho_{\sigma}(\mathbf{r})} \sum_{j=1}^{N_{\sigma}}\left|\varphi_{j \sigma}(\mathbf{r})\right|^{2} u_{x j \sigma}(\mathbf{r})+c . c .
$$

is the so-called Slater potential;

$$
\Delta v_{x \sigma}^{\mathrm{KLI}}(\mathbf{r})=\frac{1}{2 \rho_{\sigma}(\mathbf{r})} \sum_{j=1}^{N_{\sigma}}\left|\varphi_{j \sigma}(\mathbf{r})\right|^{2}\left(\bar{v}_{x j \sigma}-\bar{u}_{x j \sigma}\right)+c . c .
$$

is the correction to the Slater potential added by the KLI approximation of the OEP solution; and

$$
\Delta v_{x \sigma}^{\mathrm{OS}}(\mathbf{r})=\frac{-1}{2 \rho_{\sigma}(\mathbf{r})} \sum_{j=1}^{N_{\sigma}}\left|\varphi_{j \sigma}(\mathbf{r})\right|^{2} \nabla \cdot\left(\psi_{j \sigma}^{*}(\mathbf{r}) \nabla \varphi_{j \sigma}(\mathbf{r})\right)+c . c .
$$

is the correction to the KLI solution, which adding the contribution from the orbital shifts, provide the full OEP solution. Thus, the definition of $\Delta v_{x \sigma}^{\mathrm{OEP}}(\mathbf{r})$ in Eq. (18) becomes clear. We remind that the asymptotic limit of $v_{x \sigma}(\mathbf{r})$ in Eq. (18), for finite systems, is given by the first term, $v_{x \sigma}^{\mathrm{SL}}(r \rightarrow \infty) \rightarrow-1 / r: 14,30$

We point out that the derivations above are fully independent of the dimensionality. In orbital functionals the dimensional character is ensured from the structure of the KS orbitals themselves, and no further assumptions are made. Therefore, the OEP (and KLI) schemes presented above apply equally well to $3 \mathrm{D}$ and $2 \mathrm{D}$. Below, however, we focus on a recent approximation to $\Delta v_{x \sigma}^{\mathrm{OEP}}$ proposed by Becke and Johnson,, 25 (BJ) which is dimensiondependent by construction. In $3 \mathrm{D}$, the $\mathrm{BJ}$ approximation has received considerable attention, $\underline{42,43,44,45,46,47,48,49}$

\section{BECKE-JOHNSON APPROXIMATION}

\section{A. Three dimensions}

For 3D systems, Becke and Johnson have proposed to approximate $\Delta v_{x \sigma}^{\mathrm{OEP}}(\mathbf{r})$ by $\Delta v_{x \sigma}^{\mathrm{BJ}}(\mathbf{r})$, defined $\mathrm{as}^{25}$

$$
\Delta v_{x \sigma}^{\mathrm{BJ}}(\mathbf{r})=\frac{1}{\pi}\left(\frac{5}{12}\right)^{1 / 2} \sqrt{\left[\frac{\tau_{\sigma}(\mathbf{r})}{\rho_{\sigma}(\mathbf{r})}\right]}
$$

with

$$
\tau_{\sigma}(\mathbf{r})=\sum_{k=1}^{N_{\sigma}}\left|\nabla \varphi_{k \sigma}(\mathbf{r})\right|^{2},
$$

being (twice) the spin-dependent kinetic-energy density. This approximation was found by seeking a simple expression having the following properties: (i) it is (possibly) invariant with respect to unitary orbital transformation; (ii) it provides an exact treatment of any (ground state) hydrogenic atom; (iii) it has a step-like structure characteristic of $\Delta v_{x \sigma}^{\mathrm{OEP}}(\mathbf{r})$ in multi-shell atoms; $28,29,30$ (iv) it is exact in the $3 \mathrm{D}$ uniform electron gas limit, $\Delta v_{x \sigma}^{\mathrm{OEP}}=\Delta v_{x \sigma}^{\mathrm{BJ}}=\left[3 \rho_{\sigma} /(4 \pi)\right]^{1 / 3}$, with constant spin densities. The BJ potential as defined in Eq. (22) has been a

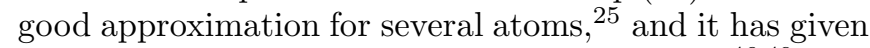
valid estimations for semiconductor band gaps $\underline{46,49}$ 


\section{B. Extension to two dimensions}

In the framework of the BJ approach, we may suggest the following approximation to be valid in $2 \mathrm{D}$,

$$
\Delta v_{x \sigma}(\mathbf{r})=\frac{2 \sqrt{2}}{3 \pi} \sqrt{\left[\frac{\tau_{\sigma}(\mathbf{r})}{\rho_{\sigma}(\mathbf{r})}\right]}
$$

where the coefficient $2 \sqrt{2} /(3 \pi)$ is chosen to satisfy the exact $2 \mathrm{D}$ uniform electron gas limit

$$
\Delta v_{x \sigma}^{\mathrm{OEP}}=\Delta v_{x \sigma}=\frac{4}{3 \sqrt{\pi}} \rho_{\sigma}^{1 / 2}
$$

with constant densities. However, as shown analytically and also numerically in the next section, this form serves only as the starting point. We need to introduce two completely general modifications, based on an additional set of physical requirements.

First, we observe that property (i) given in the previous section is satisfied only under the restriction of real-valued orbitals. In fact, it is easy to see that under gauge transformation for both real and complex-valued orbitals the BJ contribution to the exchange potential is not gauge invariant. The relevance of the gauge invariance requirement for the meta-generalized-gradient approximation has been analyzed in some detail by Tao and Perdew 50 We expect that the same criterion should apply to any approximation for $v_{x \sigma}(\mathbf{r})$ which depend explicitly on $\tau_{\sigma}(\mathbf{r})$. We can ensure the gauge invariance by replacing $\tau_{\sigma}(\mathbf{r})$ with 19,50

$$
\tau_{\sigma}(\mathbf{r}) \longrightarrow \bar{\tau}_{\sigma}(\mathbf{r})=\tau_{\sigma}(\mathbf{r})-\frac{\mathbf{j}_{p \sigma}^{2}(\mathbf{r})}{\rho_{\sigma}(\mathbf{r})}
$$

where

$$
\mathbf{j}_{p \sigma}(\mathbf{r})=\frac{1}{2 i} \sum_{k=1}^{N_{\sigma}}\left[\varphi_{k \sigma}^{*}(\mathbf{r})\left(\nabla \varphi_{k \sigma}(\mathbf{r})\right)-\left(\nabla \varphi_{k \sigma}^{*}(\mathbf{r})\right) \varphi_{k \sigma}(\mathbf{r})\right]
$$

is the spin-dependent paramagnetic current density.

Second, property (ii) given in the previous section is not valid for all one-electron systems. As we show below, this also relates to the wrong (divergent) behavior of the approximation in the asymptotic region. As it has been shown in 3D, an ad-hoc solution may be found ${ }^{42}$ for specific cases, but still it would be preferable to have a general cure. Hence, to obtain an expression which vanishes in the case of a generic single-particle state, we add an additional term

$$
\bar{\tau}_{\sigma}(\mathbf{r}) \longrightarrow D_{\sigma}(\mathbf{r})=\bar{\tau}_{\sigma}(\mathbf{r})-\frac{1}{4} \frac{\left(\nabla \rho_{\sigma}(\mathbf{r})\right)^{2}}{\rho_{\sigma}(\mathbf{r})}
$$

In conclusion, in $2 \mathrm{D}$ we obtain

$$
\Delta v_{x \sigma}(\mathbf{r}) \longrightarrow \Delta v_{x \sigma}^{\mathrm{C}}(\mathbf{r})=\frac{2 \sqrt{2}}{3 \pi} \sqrt{\left[\frac{D_{\sigma}(\mathbf{r})}{\rho_{\sigma}(\mathbf{r})}\right]}
$$

The present approximation for the total corrected $2 \mathrm{D}$ exchange potential reads then

$$
\begin{aligned}
v_{x \sigma}(\mathbf{r}) & =v_{x \sigma}^{\mathrm{SL}}(\mathbf{r})+\Delta v_{x \sigma}^{\mathrm{C}}(\mathbf{r}) \\
& =v_{x \sigma}^{\mathrm{SL}}(\mathbf{r})+\frac{2 \sqrt{2}}{3 \pi} \sqrt{\left[\frac{D_{\sigma}(\mathbf{r})}{\rho_{\sigma}(\mathbf{r})}\right]}
\end{aligned}
$$

with

$$
D_{\sigma}(\mathbf{r})=\tau_{\sigma}(\mathbf{r})-\frac{1}{4} \frac{\left(\nabla \rho_{\sigma}(\mathbf{r})\right)^{2}}{\rho_{\sigma}(\mathbf{r})}-\frac{\mathbf{j}_{p \sigma}^{2}(\mathbf{r})}{\rho_{\sigma}(\mathbf{r})} .
$$

Eq. (30) is the key expression of the present work, and in the following we will discuss its properties, first analytically and then numerically. First, it is easy to see that $D_{\sigma}(\mathbf{r}) \equiv 0$ for any one-electron system (per spin channel), as it should be [property (i)]. To see this, it is enough to note that for this particular case $\rho_{\sigma}(r)=\left|\varphi_{\sigma}(r)\right|^{2}$, with $\varphi_{\sigma}(r)=\sqrt{\rho_{\sigma}(r)} e^{i \theta(r)}$, with no loss of generality. Substituting this in Eq. (31), the result $D_{\sigma}(\mathbf{r}) \equiv 0$ follows at once, for arbitrary functions $\rho_{\sigma}(\mathbf{r})$ and $\theta(\mathbf{r})$. Second, in the asymptotic limit, $D_{\sigma}(r \rightarrow \infty) \rightarrow 0$, for any finite system. To see this, it is enough to note that for any finite system, all ingredients of $D_{\sigma}(\mathbf{r})$ in Eq. (31) become dominated by the contribution of the highest occupied orbital. As a consequence, in the asymptotic region the system becomes effectively one-electron like, and the result $D_{\sigma}(r \rightarrow \infty) \rightarrow 0$ applies. This guarantees that $v_{x \sigma}(\mathbf{r})$ has the correct asymptotic behavior given by $v_{x \sigma}^{\mathrm{SL}}(\mathbf{r})$. Besides correcting the potential in the asymptotic region, we also expect improvements at finite r. We find this reasonable due to the fact that $D_{\sigma}(\mathbf{r})$ is the main ingredient of the so-called electron localization function, $56,57,58$ which is able to deal with situations where the multi-shell structure of atoms becomes relevant, as well as in the region of interest for chemical bonding.

Then, it is reassuring to note that one can justify the choice of the power $\alpha$ in

$$
\Delta v_{x \sigma}^{\mathrm{C}}(\mathbf{r})=\frac{2 \sqrt{2}}{3 \pi}\left[\frac{D_{\sigma}(\mathbf{r})}{\rho_{\sigma}(\mathbf{r})}\right]^{\alpha},
$$

by observing that the resulting potential scales linearly only for $\alpha=1 / 2$, similarly to the exact exchange potential. 51 Under uniform scaling of the coordinates, $\mathbf{r} \rightarrow \lambda \mathbf{r}$ (with $0<\lambda<\infty$ ), and for the norm-preserving many-body wave function, the $2 \mathrm{D}$ density scales quadratically with $\lambda, \rho_{\sigma}^{\lambda}(\mathbf{r}) \rightarrow \lambda^{2} \rho_{\sigma}(\lambda \mathbf{r})$. This leads to the result that the KS orbitals in $2 \mathrm{D}$ are seen to scale as $\varphi_{k \sigma}^{\lambda}(\mathbf{r}) \rightarrow \lambda \varphi_{k \sigma}(\lambda \mathbf{r})$. Thus, $\tau_{\sigma}^{\lambda}(\mathbf{r}) \rightarrow \lambda^{4} \tau_{\sigma}(\lambda \mathbf{r})$, $\nabla \rho_{\sigma}^{\lambda}(\mathbf{r}) \rightarrow \lambda^{3} \nabla_{\lambda \mathbf{r}} \rho_{\sigma}(\lambda \mathbf{r})$, and $\mathbf{j}_{p \sigma}^{\lambda}(\mathbf{r}) \rightarrow \lambda^{3} \mathbf{j}_{p \sigma}(\lambda \mathbf{r})$. Substituting these relations to Eq. (32) yields the scaling relation

$$
\Delta v_{x \sigma}^{\mathrm{C} \lambda}(\mathbf{r}) \rightarrow \lambda^{2 \alpha} \Delta v_{x \sigma}^{\mathrm{C}}(\lambda \mathbf{r})
$$

which fulfills the exact linear scaling constraint only if $\alpha=1 / 2$. 
Finally, we remind that the calculation of $\Delta v_{x \sigma}^{\mathrm{C}}(\mathbf{r})$ is computationally cheap in comparison with the full OEP method or the KLI approximation, which is naturally the case also for the conventional $\Delta v_{x \sigma}^{\mathrm{BJ}}(\mathbf{r})$. The calculation of the Slater part of the total potential is actually still costly. For this part, we have previously provided accurate and numerically simple approximations in $2 \mathrm{D}$ by considering the properties of the exchange hole, $\underline{13,14}$

\section{RESULTS}

\section{A. Single-electron states of a harmonic oscillator}

First, we consider the single-electron non-interacting eigenstates in a $2 \mathrm{D}$ isotropic harmonic oscillator. The external confining potential is given by $v_{0 \sigma}(r)=\omega^{2} r^{2} / 2$, where $\omega$ is the oscillator strength. The use of this system is motivated by the frequent use of such a confining potential when modeling $2 \mathrm{D}$ quantum dots $\underline{4}$ (see also below). The eigenstates, with the associated densities, are given by

$$
\begin{aligned}
\phi_{n l}(r, \theta) & =f_{n l}(r) e^{-r^{2} / 2} e^{i l \theta}, \\
\rho_{n l}(r) & =\left|\phi_{n l}(r, \theta)\right|^{2}=f_{n l}^{2}(r) e^{-r^{2}},
\end{aligned}
$$

respectively. Here $r$ and $\theta$ are the usual radial and angular 2D polar coordinates. $n=0,1,2, \ldots$ and $l=$ $0, \pm 1, \pm 2, \ldots$ are the radial and angular quantum numbers, and $f_{n l}(r)$ is a radial function, related to Laguerre polynomials. For simplicity, we do not consider the spin index in this analysis. The corresponding contribution to the kinetic energy density is given by

$$
\begin{aligned}
\tau_{n l}(r)=\left|\nabla \phi_{n l}(r, \theta)\right|^{2} & =\left[\frac{d f_{n l}(r)}{d r}-r f_{n l}(r)\right]^{2} e^{-r^{2}} \\
& +\frac{l^{2} \rho_{n l}(r)}{r^{2}} .
\end{aligned}
$$

Following Eq. (24), we obtain

$$
\frac{\tau_{n l}(r)}{\rho_{n l}(r)}=\left[\frac{d f_{n l}(r) / d r}{f_{n l}(r)}-r\right]^{2}+\frac{l^{2}}{r^{2}} .
$$

Clearly, this contribution to $\Delta v_{x \sigma}(\mathbf{r})$ diverges linearly for $r \rightarrow \infty$, after summing over all occupied states (all showing the same divergent behavior), and after taking the square root of the sum. On the other hand, this divergent behavior is absent in our corrected exchange potential in Eq. (30), as it cancels exactly with the second term of $D_{\sigma}(\mathbf{r})$ in Eq. (31).

\section{B. Many-electron quantum dots}

Next we focus on the same system as in the previous section but consider $N$ interacting electrons. Within

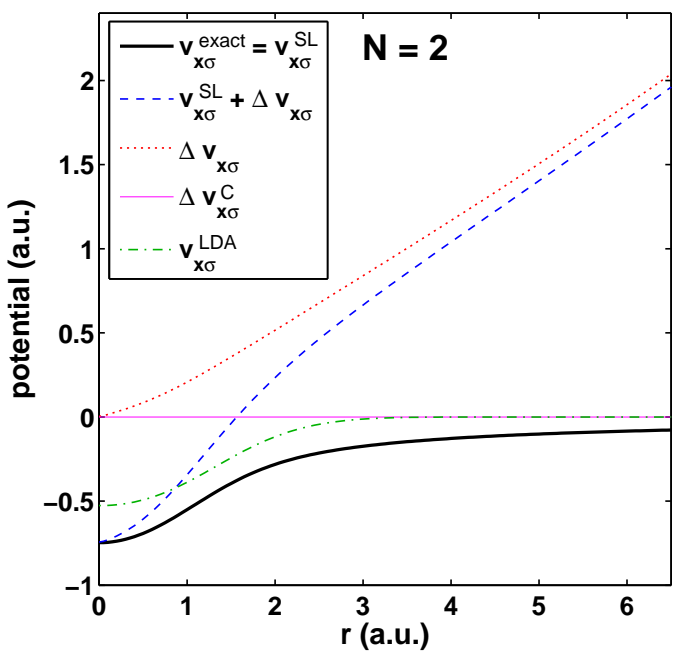

FIG. 1: (Color online) Exchange potentials for a two-electron quantum dot (singlet). The results are calculated from the analytic density. $\Delta v_{x \sigma}$ and $\Delta v_{x \sigma}^{\mathrm{C}}$ are defined in Eqs. (24) and (29), respectively.

the effective-mass approximation for electrons in semiconducting host material such as GaAs, this is the most common model for $2 \mathrm{D}$ quantum dots $\underline{\underline{4}}$

First, we consider the singlet solution of $N=2$ and $\omega=1$ a.u., which is known analytically $\underline{52}$ In this case, the exact exchange potential is equal to the Slater potential (and minus half of the Hartree potential). Therefore, any correction to the Slater potential should be zero. For the kinetic-energy density we find $\tau_{\sigma}(\mathbf{r})=\left(\nabla \rho_{\sigma}(\mathbf{r})\right)^{2} /\left(4 \rho_{\sigma}(\mathbf{r})\right)$, and the non-corrected Becke-Johnson-type approximation in Eq. (24) becomes $\Delta v_{x \sigma}(\mathbf{r})=\sqrt{2} \nabla \rho_{\sigma}(\mathbf{r}) /\left(3 \pi \rho_{\sigma}(\mathbf{r})\right)$, where the spin density has an analytic expression

$$
\begin{aligned}
\rho_{\sigma}(r) & =\frac{2}{\pi(\sqrt{2 \pi}+3)}\left\{e^{-r^{2}}\left(1+r^{2} / 2\right)+\frac{1}{2} \sqrt{\pi} e^{-3 r^{2} / 2}\right. \\
& \left.\times\left[I_{0}\left(r^{2} / 2\right)+r^{2} I_{0}\left(r^{2} / 2\right)+r^{2} I_{1}\left(r^{2} / 2\right)\right]\right\},
\end{aligned}
$$

deduced from the analytic wave function 52 Here $I_{0}$ and $I_{1}$ are the zeroth and first-order modified Bessel functions of the first kind, respectively. Figure 1 shows that $\Delta v_{x \sigma}(\mathbf{r})$ (dotted line) has clear divergent behavior, making the total exchange potential (dashed line) largely different from the exact result (thick solid line), even in the small- $r$ regime. It should be noted that also the LDA exchange potential considerably deviates from the exact result. On the other hand, our corrected scheme in Eq. (30) yields the exact result: $\Delta v_{x \sigma}^{\mathrm{C}}(\mathbf{r})=0$ as discussed above.

The divergent behavior of $\Delta v_{x \sigma}(r)$ may be easily extracted from the interacting density given in Eq. (38). For this, one should use the asymptotic expansion of the modified Bessel functions: $I_{\nu}(z \rightarrow \infty) \rightarrow e^{z} / \sqrt{2 \pi z}+\ldots$ (Ref. 53). Using this in Eq. (38) leads to $\rho_{\sigma}(r) \rightarrow$ $r^{2} e^{-r^{2}} / \pi(3+\sqrt{2 \pi})$, and $\nabla \rho_{\sigma}(r) \rightarrow-2 r^{3} e^{-r^{2}} / \pi(3+\sqrt{2 \pi})$ in the asymptotic region $(r \rightarrow \infty)$. Replacing everything, 


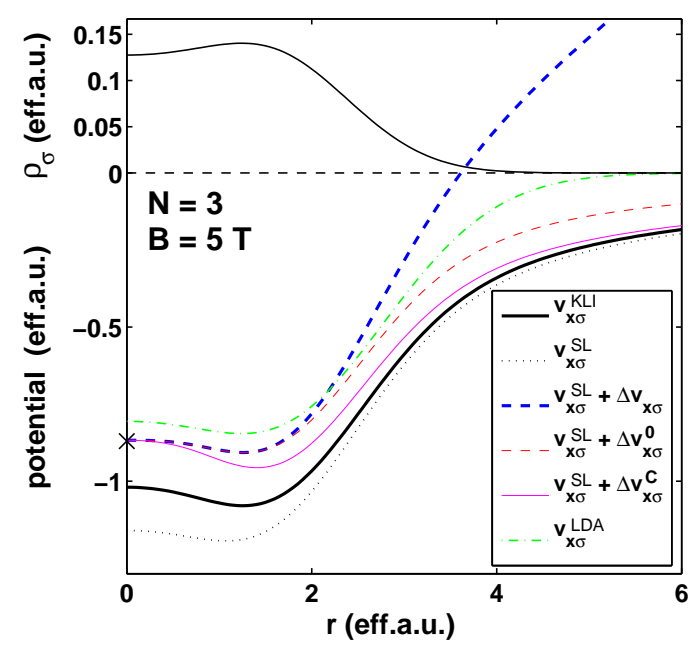

FIG. 2: (Color online) Exchange potentials for a fully spinpolarized three-electron quantum dot in external magnetic field of $B=5 \mathrm{~T}$. Terms with $\Delta v_{x \sigma}^{\mathrm{C}}$ and $\Delta v_{x \sigma}^{0}$ refer to the corrected approximation [Eq. [30] ], with and without the explicit current term in Eq. (31), respectively. The scales for the vertical axis referring to the potentials and density (negative and positive values, respectively) are different.

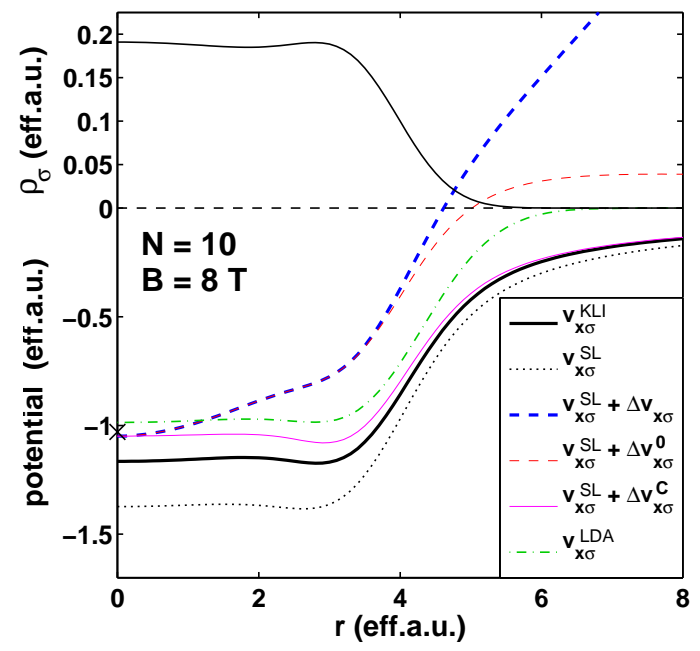

FIG. 3: (Color online) Same as Fig. 2 but for ten electrons at $B=8 \mathrm{~T}$.

we obtain that $\Delta v_{x \sigma}(r \rightarrow \infty) \rightarrow 2 \sqrt{2} r /(3 \pi) \simeq 0.300 r$ in agreement with Fig. 1. It is interesting to note that the leading term to the divergent contribution obtained for the interacting system agrees exactly with the noninteracting estimate given above in Eq. (37). The reason is that the density is rather small in the asymptotic region, and then the modifications introduced by interactions to the bare 2D harmonic potential are also rather small. As a consequence, the interacting densities approach the non-interacting limit asymptotically.

In Figs. 2 and 3 we show the exchange potentials $v_{x \sigma}$ for quantum dots containing three and ten interacting electrons, respectively. In both cases the oscillator strength is $\omega=0.4217$ in effective atomic units (eff.a.u.) with the material parameters for GaAs: $m^{*}=0.067 m_{e}$ and $\epsilon=12.4 \epsilon_{0}$. Here we have performed self-consistent KLI calculations by applying the octopus DFT code,,$\underline{54}$ and use the resulting KS orbitals as inputs in the approximations given above. Both dots are exposed to an external, perpendicular magnetic field with a sufficient strength to achieve full spin polarization $(S=N / 2)$ and occupation to eigenstates of consecutive angular momenta from $l=0$ to $l=-N+1$. The resulting "maximum-density droplet" $\underline{55}$ has a smooth electron density (see the upper panels of Figs. 2 and 3). The corresponding exchange potentials calculated with different approximations have several interesting features listed below.

- Similarly to the previous examples, the straightforward BJ extension, i.e., Slater potential combined with $\Delta v_{x \sigma}$ in Eq. (24), shows divergent behavior.

- Our approximation $\Delta v_{x \sigma}^{\mathrm{C}}(\mathbf{r})$ in Eq. (30) leads to good overall agreement with the KLI potential, especially at large $r$. The agreement improves as a function of $N$.

- The LDA exchange potential is overall worse than our approximation - not only in the asymptotic region showing the obvious exponential decay - but also close to the center of the system.

- The current-dependent term introduced in Eq. (26), which enforces the gauge invariance, is crucial in finding the correct behavior. Without this term $\left(\Delta v_{x \sigma}^{0}\right)$ the performance is very poor at high magnetic fields (see in particular Fig. 3 with $B=8 \mathrm{~T})$.

- Close to the center of the maximum-density droplet, which locally resembles the $2 \mathrm{DEG}$ with the electron localization function $\approx 1 / 2$ (Ref. 58 ), the present approximation satisfies with a good precision the well-known exact relation for the homogeneous 2DEG between the exchange potential and the Slater potential: $v_{x \sigma}=(3 / 4) v_{x \sigma}^{\text {SL }}$ (see the crosses in Figs. 2] and 3). For the calculation of the cross positions, the numerical value of $v_{x \sigma}^{\mathrm{SL}}(r=0)$ has been multiplied by $3 / 4$. Note that this relation is not well satisfied between $v_{x \sigma}^{\mathrm{LDA}}(r=0)$ and $v_{x \sigma}^{\mathrm{SL}}(r=0)$, because the quantum-dot system is finite, and globally not homogeneous.

Figure 4 shows the exchange potentials for a closedshell quantum dot with 20 electrons. Again, the straightforward BJ extension to 2D diverges, whereas the cured approximation qualitatively agrees with the KLI solution. It should be noted that the shell structure is better reproduced by the present approximation than by the LDA. In this system, the (3/4)-relation is not well satisfied (see the cross in Fig. (4), since the system properties in the core region are far from those of the homogeneous 2DEG, i.e., the electron localization function is 


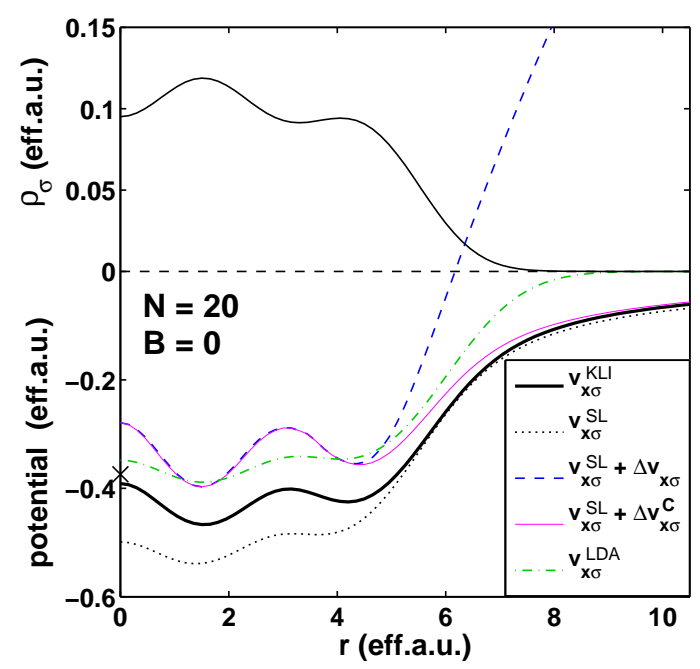

FIG. 4: (Color online) Exchange potentials for a closed-shell quantum dot with 20 electrons.

varying. 58 Also note that for this closed-shell system, the spin-dependent paramagnetic current density vanishes, and then $\Delta v_{x \sigma}^{\mathrm{C}}(\mathbf{r})=\Delta v_{x \sigma}^{0}(\mathbf{r})$.

Finally, we point out that the present scheme, in particular the modifications introduced in Eqs. (26) and (28), can be considered and reworked also in 3D. This has been shown to lead to an approximation for the 3D exchange potential that gives good results in systems possessing orbital currents (e.g., open-shell systems), in the presence of external magnetic or electric fields, or in "non-atomic" systems such as the Hooke's atom, i.e., the
$3 \mathrm{D}$ counterpart of the harmonic quantum dot $\underline{59}$

\section{CONCLUSIONS}

To achieve an accurate and computationally convenient approximation to the exchange potential in two dimensions, we have applied and extended the formalism of Becke and Johnson commonly used in three dimensions. We have found that direct extension of the formalism leads to divergent behavior of the exchange potential in the most standard two-dimensional applications. We have corrected the scheme by introducing a current-dependent term ensuring the gauge invariance and a density-gradient term capturing the disappearance of the correction to the Slater contribution for a generic single-particle state. The resulting approximation for the exchange potential agrees well with the Krieger-Li-Iafrate potential in various quantum dots up to high magnetic fields and thus to high current densities. The correct asymptotic behavior is recovered and the shell-structure is well described. Similar strategy is expected to lead to good results also in three-dimensional applications in external magnetic and/or electric fields.

\section{Acknowledgments}

This work was supported by the Deutsche Forschungsgemeinschaft and the Academy of Finland. C.R.P. was supported by the European Community through a Marie Curie IIF (Grant No. MIF1-CT-2006-040222) and CONICET of Argentina through Grant No. PIP 5254.
* Electronic address: pittaliss@missouri.edu

† Electronic address: erasanen@jyu.fi

¥ Permanent address: Centro Atómico Bariloche and Instituto Balseiro, 8400 S.C. de Bariloche, Río Negro, Argentina

1 R. M. Dreizler and E. K. U. Gross, Density Functional Theory (Springer, Berlin, 1990).

2 U. von Barth, Phys. Scr. T109, 9 (2004).

3 J. P. Perdew and S. Kurth, in A Primer in Density Functional Theory, Lecture Notes in Physics Vol. 620, edited by C. Fiolhais, F. Nogueira, and M. Marques (Springer, Berlin, 2003).

${ }^{4}$ L. P. Kouwenhoven, D. G. Austing, and S. Tarucha, Rep. Prog. Phys. 64, 701 (2001); S. M. Reimann and M. Manninen, Rev. Mod. Phys. 74, 1283 (2002).

5 Y.-H. Kim, I.-H. Lee, S. Nagaraja, J.-P. Leburton, R. Q. Hood, and R. M. Martin, Phys. Rev. B 61, 5202 (2000).

${ }^{6}$ L. Pollack and J. P. Perdew, J. Phys.: Condens. Matter 12, 1239 (2000).

7 L. A. Constantin, Phys. Rev. B 78, 155106 (2008).

8 A. K. Rajagopal and J. C. Kimball, Phys. Rev. B 15, 2819 (1977).

9 B. Tanatar, D. M. Ceperley, Phys. Rev. B 39, 5005 (1989).

10 C. Attaccalite, S. Moroni, P. Gori-Giorgi, and G. B.
Bachelet, Phys. Rev. Lett. 88, 256601 (2002).

11 H. Saarikoski, E. Räsänen, S. Siljamäki, A. Harju, M. J. Puska, and R. M. Nieminen, Phys. Rev. B 67, 205327 (2003).

12 L. A. Constantin, J. P. Perdew, and J. M. Pitarke, Phys. Rev. Lett. 101, 016406 (2008). See also erratum.

13 S. Pittalis, E. Räsänen, N. Helbig, and E. K. U. Gross, Phys. Rev. B 76, 235314 (2007).

14 E. Räsänen, S. Pittalis, C. R. Proetto, and E. K. U. Gross, Phys. Rev. B 79, 121305(R) (2009).

15 N. Helbig, S. Kurth, S. Pittalis, E. Räsänen, and E. K. U. Gross, Phys. Rev. B 77, 245106 (2008).

16 S. Pittalis, E. Räsänen, and M. A. L. Marques, Phys. Rev. B 78, 195322 (2008).

17 S. Pittalis, E. Räsänen, J. G. Vilhena, and M. A. L. Marques, Phys. Rev. A 79, 012503 (2009).

18 S. Pittalis, E. Räsänen, C. R. Proetto, and E. K. U. Gross, Phys. Rev. B 79, 085316 (2009).

19 S. Pittalis, E. Räsänen, and E. K. U. Gross, Phys. Rev. A 80, 032515 (2009).

20 S. Rigamonti and C. R. Proetto, Phys. Rev. Lett. 98, 066806 (2007).

21 P. Gori-Giorgi, M. Seidl, and G. Vignale, submitted, arXiv: 0908.0669 . 
22 R. Sharp and G. Horton, Phys. Rev. 90, 317 (1953).

23 J. D. Talman and W. F. Shadwick, Phys. Rev. A 14, 36 (1976).

24 J. B. Krieger, Y. Li, and G. J. Iafrate, Phys. Rev. A 45, 101 (1992).

${ }^{25}$ A. D. Becke and E. R. Johnson, J. Chem. Phys. 124, 221101 (2006).

26 U. von Barth, Phys. Rev. A 20, 1693 (1979).

27 U. von Barth and L. Hedin, J. Phys. C 5, 1629 (1972).

28 S. Kümmel, L. Kronik, Rev. Mod. Phys. 80, 3 (2008).

29 E. Engel, in A Primer in Density Functional Theory, Vol. 620 of Lecture Notes in Physics, edited by C. Fiolhais, F. Nogueira, and M. Marques (Springer, Berlin, 2003), p. 1.

30 T. Grabo, T. Kreibich, S. Kurth, and E.K.U. Gross, in Strong Coulomb Correlations in Electronic Structure Calculations: Beyond Local Density Approximations, edited by V. Anisimov (Gordon and Breach, Amsterdam, 2000), p. 203.

31 J. B. Krieger, Y. Li, and G. J. Iafrate, Phys. Rev. A 46, 5453 (1992).

32 S. Ivanov, S. Hirata, and R. J. Bartlett, Phys. Rev. Lett. 83, 5455 (1999).

33 A. Görling, Phys. Rev. Lett. 83, 5459 (1999).

${ }^{34}$ M. Städele, J. A. Majewski, P. Vogl, and A. Görling, Phys. Rev. Lett. 79, 2089 (1997).

35 C. M. Horowitz, C. R. Proetto, and S. Rigamonti Phys. Rev. Lett. 97, 026802 (2006).

36 S. Kümmel and J. P. Perdew, Phys. Rev. Lett. 90, 043004 (2003).

37 S. Kümmel and J. P. Perdew, Phys. Rev. B 68, 035103 (2003).

38 S. Sharma, J. K. Dewhurst, C. Ambrosch-Draxl, S. Kurth, N. Helbig, S. Pittalis, S. Shallcross, L. Nordström, and E. K. U. Gross, Phys. Rev. Lett. 98, 196405 (2007).

39 S. Sharma, S. Pittalis, S. Kurth, S. Shallcross, J. K. Dewhurst, and E. K. U. Gross, Phys. Rev. B 76, 100401(R) (2007).
40 D. Ködderitzsch, H. Ebert, and E. Engel, Phys. Rev. B 77, 045101 (2008).

41 E. Engel, D. Ködderitzsch, and H. Ebert, Phys. Rev. B 78, 235123 (2008).

42 R. Armiento, S. Kümmel, and T. Körzdörfer, Phys. Rev. B 77, 165106 (2008).

43 V. N. Staroverov, J. Chem. Phys. 129, 134103 (2008).

44 M. Kodera, K. Higuchi, A. Narita, and M. Higuchi, Phys. Rev. A 78, 012501 (2008).

45 A. P. Gaiduk and V. N. Staroverov, J. Chem. Phys. 128, 204101 (2008).

46 F. Tran, P. Blaha, and K. Schwarz, J. Phys.: Condens. Matter 19, 196208 (2007).

47 K. E. Riley, J. Vondrášek, and P. Hobza, Phys. Chem. Chem. Phys. 9, 5555 (2007).

48 N. Umezawa, Phys. Rev. A 74, 032505 (2006).

49 F. Tran and P. Blaha, Phys. Rev. Lett. 102, 226401 (2009).

50 J. Tao and J. P. Perdew, Phys. Rev. Lett. 95, 196403 (2005).

51 M. Levy and J. P. Perdew Phys. Rev. A 32, 2010 (1985).

52 M. Taut, J. Phys. A 27, 1045 (1994).

53 M. Abramowitz and I. Stegun, Handbook of Mathematical Functions (Dover, New York, 1964), p. 377.

54 M. A. L. Marques, A. Castro, G.F. Bertsch, A. Rubio, Comput. Phys. Commun. 151, 60 (2003); A. Castro, H. Appel, M. Oliveira, C. A. Rozzi, X. Andrade, F. Lorenzen, M. A. L. Marques, E. K. U. Gross, and A. Rubio, Phys. Stat. Sol. (b) 243, 2465 (2006).

55 A. H. MacDonald, S. R. Eric Yang, and M. D. Johnson, Aust. J. Phys. 46, 345 (1993).

56 A. D. Becke and K. E. Edgecombe, J. Chem. Phys. 92, 5397 (1990).

57 T. Burnus, M. A. L. Marques, and E. K. U. Gross, Phys. Rev. A 71, 010501(R) (2005).

58 E. Räsänen, A. Castro, and E. K. U. Gross, Phys. Rev. B 77, 115108 (2008).

59 E. Räsänen, S. Pittalis, and C. R. Proetto, submitted, arXiv: 0909.1477. 